Post-print version of:

Publisher: Sage

Journal paper: Journal of Vibration and Control 2018, 24(19) 4620-4629

Title: Excitation device for high frequency vibration analysis: design and test results

Authors: P. Neri

Creative Commons Attribution Non-Commercial No Derivatives License

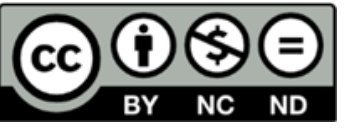

DOI Link: https://doi.org/10.1177/1077546317731210 


\title{
Excitation device for high frequency vibration analysis: design and test results
}

\author{
Paolo Neri ${ }^{1}$
}

\begin{abstract}
In the present paper, the design and optimization of a high frequency excitation source is presented. The device was developed for a harmonic response analysis test bench, aimed at dynamic characterization and resonance prediction of mechanical structures. A wide frequency range must be covered, depending on the analyzed structure: the range $1-10 \mathrm{kHz}$ was considered in the present work. The device was designed for a test bench aimed at investigating the vibrational response of centrifugal compressor bladed wheels. A really compact solution was needed since the final test bench provides one exciter for each blade (up to 20 devices on the circumference hoop). Both contact and contactless solutions were considered, but only the contact solution was found to fulfill all the specifications. Finally, different stinger solutions were proposed and compared in the paper. The investigated solutions were: a beam stinger (diameter $1 \mathrm{~mm}$ ), a wire stinger (diameter $0.2 \mathrm{~mm}$ ), and a ball stinger (diameter $3 \mathrm{~mm}$ ) with two different support solutions. Experimental tests performed on a device prototype allowed to verify the specifications fulfillment and to choose the best stinger solution for the application.
\end{abstract}

\section{Keywords}

High frequency excitation source, Electromagnet excitation, Custom electromagnetic shaker, Harmonic response analysis, Modal analysis, Bladed wheels dynamic characterization

\section{Introduction}

Structures dynamic characterization is a crucial issue in industry. When a component is subject to cyclic load excitation, a deep knowledge of its vibrational response is needed to avoid dangerous resonance conditions. In fact, resonant response could imply efficiency drop or even induce fatigue fracture failures Rieger (1990); Oberholster and Heyns (2009); Neri and Peeters (2015); Neri (2017a). Experimental Modal Analysis (EMA) and Harmonic Response Analysis (HRA) are both useful methods to evaluate the dynamic behavior of the components.

The present work focuses on the design of dynamic exciters for experimental HRA tests, which exploits single harmonic excitation. Even though the final device can be applied to any mechanical structure, bladed wheels were considered as the main application field in this research. High rotational speed machines, such as turbines and compressors, are remarkable examples of mechanical structures that experience high frequency harmonics vibrations. In this machines, the load is determined by the fluid coming from stator vanes Mukhopadhyay et al. (1998); Bertini et al. (2014b). High frequency excitation happens at the rotational speed regimes of the machine, in the range $1-10$ $\mathrm{kHz}$ Kammerer and Abhari (2009).Several papers report the analysis of this kind of components by means of EMA tests: in Bidaut and Baumann (2012); Bertini et al. (2014a) a single or double shaker EMA test is presented, while in Martarelli et al. (2011) a hammer test setup is adopted.

Anyway, EMA may not be sufficient to completely determine bladed wheels dynamic behavior, since complex shape modes need specific spacial load distribution to be properly excited according to the Singh's Advanced Frequency Evaluation (SAFE) diagram Bertini et al. (2014b,a); Singh and Vargo (1989); Singh et al. (1994); Bertini et al. (2016). This is the reason why HRA tests may be really useful, even if a complex set-up is needed. In principle, one exciter is required on each blade to have complete control on the applied load Bertini et al. (2017). This allows to reproduce any spatial load distribution on the components, so that a full investigation of the dynamic response can be achieved. Firstly, operational conditions can be reproduced (i.e. different numbers of stator vanes). Then, a comparison can be performed between different modes response levels. Finally high Number of Nodal Diameter (NND) modes can be specifically excited by imposing the needed load spatial distribution, according to the SAFE diagram. In this way an experimental validation of the method can also be obtained. These are the reasons why a dedicated test bench was designed Bertini et al. (2017) in order to operate in a wide frequency range and to control frequency, amplitude and phase of each exciter placed on a bladed wheel. Since the number of blades can be considerable with respect to the wheel diameter, the exciter lateral dimension issue represents really critical constraint for this application. Moreover, the produced load must cover the wide frequency range $1-10$

${ }^{1}$ University of Pisa, IT

\section{Corresponding author:}

Paolo Neri, University of Pisa, Departmenet of Civil and Industrial Engineering, Largo L. Lazzarino 1, Pisa, 56122, Italy.

Email: paolo.neri@dici.unipi.it 
$\mathrm{kHz}$. Both contact (electromagnetic shaker) and contactless (electromagnet) solutions were considered, in order to determine which one would provide better performances. The aforementioned excitation solutions are described in the paper and finally the chosen device is presented.

\section{Contactless solutions: magnetic exciter}

The SAFE diagram criterion highlights that a full resonance should be expected in bladed wheels when both frequency and shape matching conditions are satisfied. A proper estimation of the time varying force acting on each blade is then crucial when designing a test, especially if operational conditions need to be simulated. Contactless solutions were firstly considered, in order to avoid any influence of the excitation system on the studied component. If the bladed wheel is manufactured using ferromagnetic materials, the solicitation can be introduced by magnetic fields Maschio (2012); Battiato et al. (2017). A set of permanent magnets can be used to provide the excitation: an analytical, numerical and experimental study of this solution is presented in Neri (2013, 2017b), showing a considerable load intensity. Anyway, since these magnets provide a static magnetic field, a relative rotational speed (up to $10000 \mathrm{rpm}$ ) between the magnet set and the bladed wheel would be needed in order to have a time varying load. Safety issues and actuation problems would arise with this solution, so it was discarded. Electromagnets represent a really flexible contactless alternative, allowing to reproduce the desired spatial and temporal force distribution by simply acting on the electronic control of the device. Different electromagnet solutions are available in literature Pesek et al. (2011); Jones and Cross (2003); Berruti et al. (2011); Firrone and Berruti (2012); Firrone et al. (2013). In particular, Berruti et al. (2011); Firrone and Berruti (2012); Firrone et al. (2013) show an application of some electromagnets used to excite a bladed wheel. This solution was really attractive because it allows to precisely control frequency, phase and amplitude of each exciter using electronic equipment. Moreover, it can be proven that the frequency of the mechanical quantities (e.g. applied force) theoretically has a frequency which is double the frequency of the electric quantities (e.g. current). Since the test bench needs to work in a wide frequency range, this peculiarity would allow to reduce the working range of the electronic devices involved in the test, reducing their cost and the needed power. In the following the design and the experimental characterization of a U-shaped electromagnet is presented in order to evaluate its effectiveness for the considered application.

\section{Electromagnet modeling}

A schematic view of the electromagnet is reported in Fig.1(a), where $N$ represents the number of copper coils belonging to each winding, and $S$ represents the surface of the core which is faced to the ferromagnetic target. $R_{\mathrm{c} 1}$ and $R_{\mathrm{c} 2}$ are the reluctance of the sections of the Ushaped electromagnet core, while $R_{\mathrm{g}}$ is the reluctance of the air gap of length $l$ between the electromagnet and the target. Finally, $R_{\mathrm{t}}$ represents the reluctance of the target. The design was obtained in analogy with the device described in
Firrone and Berruti (2012). The device was modeled using the magnetic circuit method, Fig.1(b), where $i$ represents the current intensity flowing in the windings.

The reluctance of each component can be computed using the equation:

$$
R=\frac{d}{\mu S}
$$

where $d$ is the length of the component and $S$ is the surface, while $\mu$ is the permeability of the material. Since the permeability of a ferromagnetic material (like the material of the core and of the target) is much higher than the permeability of air, it is possible to state that $R_{\mathrm{c} 1} \cong R_{\mathrm{c} 2} \cong$ $R_{\mathrm{t}}<<R_{\mathrm{g}}$, so that the magnetic circuit reported in Fig.1(b) can be simplified as shown in Fig.1(c). Since the device is powered with a sinusoidal current of the form $I_{\max } \sin (\omega t+$ $\phi)$, the force acting on the target can be estimated as follows Firrone and Berruti (2012):

$$
F=\frac{(N i)^{2} \mu_{\mathrm{air}} S}{d^{2}}=\frac{N^{2} \mu_{\mathrm{air}} S}{d^{2}} \frac{I_{\mathrm{max}}^{2}}{2}(1-\cos (2 \omega t+2 \phi))
$$

proving that the force acting on the target should theoretically have twice the frequency of the sinusoidal current used to power the device.

\section{Experimental test}

The simple model described above allowed to chose the winding parameters of the electromagnet needed for the application. A prototype of the electromagnet was then built using U-shaped ferromagnetic sheets to reduce eddy current effects in the device core. Fifty coils were then winded on each arm of the electromagnet, using a wire diameter of $2 \mathrm{~mm}$. This prototype was then tested on a simplified test bench: the electromagnet was held vertically by a support to face a ferromagnetic target mounted on a load cell. This allowed to measure the force acting on the target for each working condition. Also current and voltage powering the electromagnet were measured to check the electric properties of the device. The range $2-3000 \mathrm{~Hz}$ for the electric quantities was investigated (corresponding to $4-6000 \mathrm{~Hz}$ for the mechanical quantities). Some results are reported in Fig.2, where measured voltage and force are plotted versus time for two different frequencies in the studied frequency range (a peak-to-peak current of $5 \mathrm{~A}$ was applied). Fig.2(a) shows the results of a test conducted at $800 \mathrm{~Hz}$ for the electric quantities. As can be noted, only one harmonic component at $1600 \mathrm{~Hz}$ was present in the force signal during the test, having a frequency which is the double of the voltage frequency as predicted by Eq.2. Fig.2(b) instead shows the results of a test performed with a $1400 \mathrm{~Hz}$ control signal. The force clearly presents several undesired harmonic components summed to the desired $2800 \mathrm{~Hz}$ component.

Since electric quantities present a linear behavior (having the current only one harmonic component at the same frequency of the control voltage), electromagnetic core non-linearity was excluded. Eddy current phenomena in the ferromagnetic target could be addressed as a cause of this phenomenon. The force may contain several other components besides the desired one. These components may not be relevant in force 


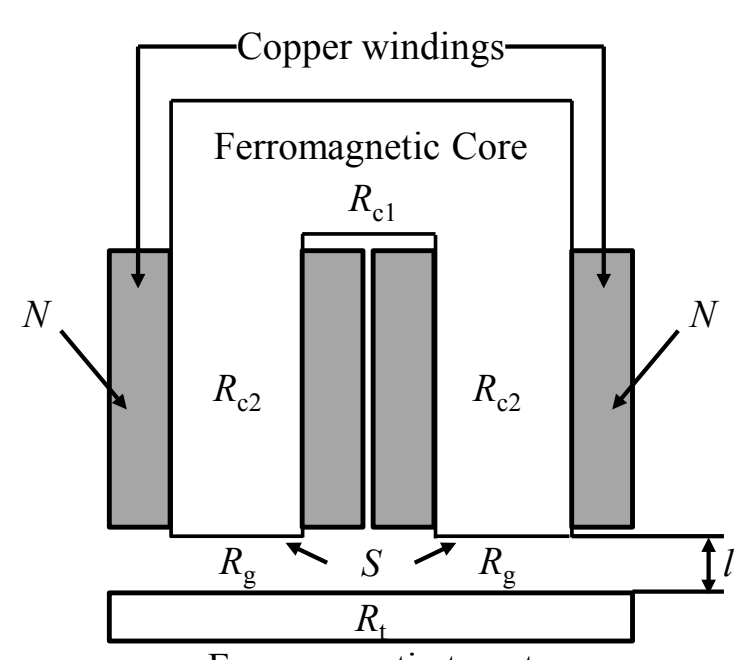

Ferromagnetic target

(a)

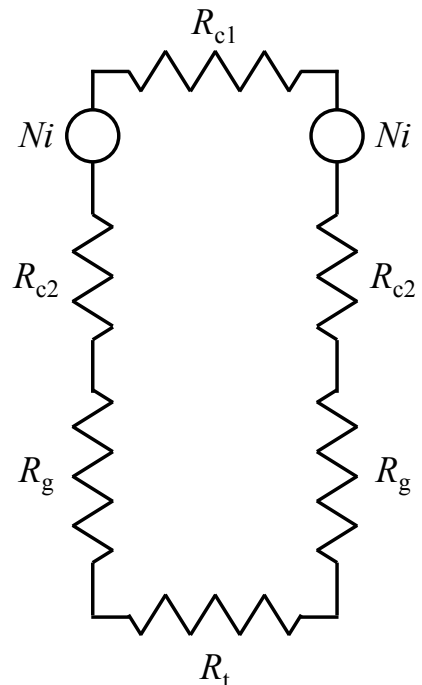

(b)

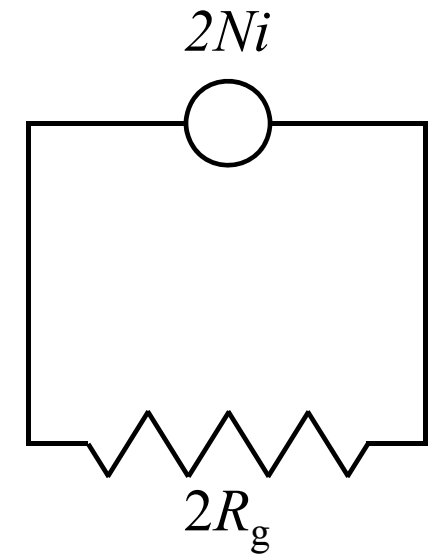

(c)

Figure 1. Model schemes: (a) electromagnet; (b) magnetic circuit; (c) simplified magnetic circuit.

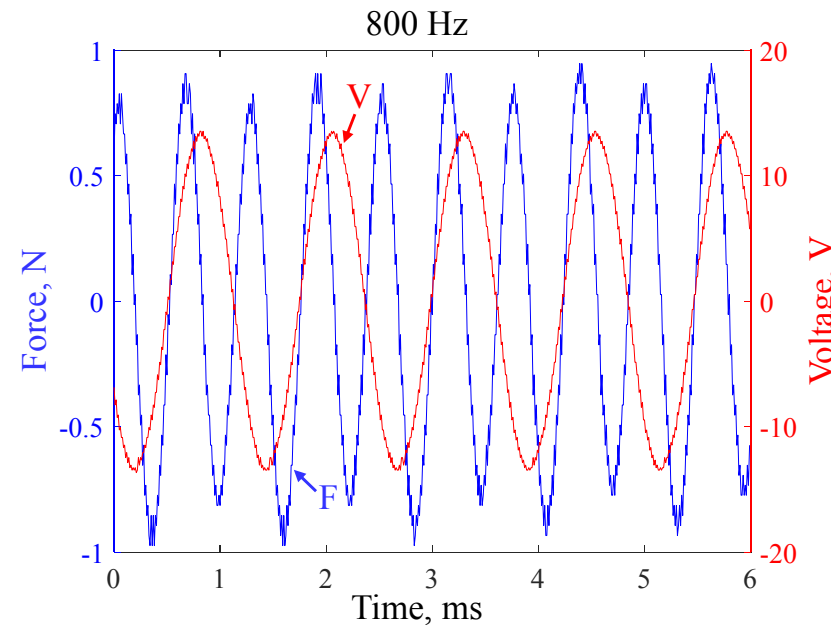

(a)

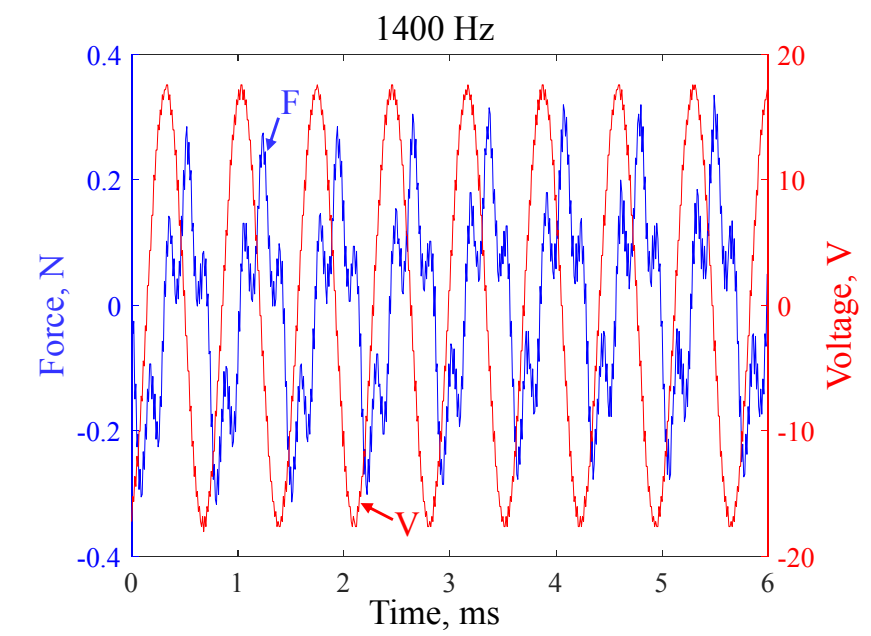

(b)

Figure 2. Electromagnet test results for two frequencies of the electric quantities (I peak-to-peak: $5 \mathrm{~A}$ ): (a) $800 \mathrm{~Hz}$; (b) $1400 \mathrm{~Hz}$.

measurement if they do not match any resonance frequency of the structure, as happens for the $800 \mathrm{~Hz}$ test. If some load harmonics match a resonance frequency instead, they could be dominant in force measurement, hiding the desired harmonic contribution. These experimental tests performed on the prototype showed severe limitations of the device. Firstly, since it is a contactless method, it is not possible to directly measure the force acting on each blade. It would be possible to measure the reaction force acting between the electromagnet and its support, which may be related to the force acting on the blade through a dynamic characterization of the electromagnet. Anyway, this procedure may result in a low force estimation accuracy, which would not allow to precisely control the force exerted on the bladed wheel. Furthermore, no good control in the harmonic content of the produced force could be achieved, thus causing control issues during HRA, which has to be conducted with a single excitation harmonic at a time. Moreover, as the working frequency increases, it was found that really high electric power was needed to produce really low peak force on the target. Finally, this contactless method could be only applied to ferromagnetic components, thus the test bench could not be used for non-ferromagnetic structures. These considerations showed that the electromagnet was not suitable for the studied application.

\section{Contact solution: electromagnetic shaker}

Since contactless solutions were found to be not suitable for the considered application, a classic electromagnetic contact shaker solution was considered. Commercial devices were not satisfactory by means of working frequency range or geometrical dimensions, so a custom exciter was designed. The device was obtained by wiring a copper coil on a support axially aligned with a moving permanent magnet, which transmits the load to the target structure. Geometry optimization was achieved by developing analytical and numerical models, so relevant force levels could be achieved in the whole frequency range, while still respecting the dimensional limitations.

Obviously, the main drawback of a contact solution is the possible influence of the device on the component response. 
The attachment between the excitation source and the structure under test may affect the dynamic behavior of the component Ewins (2000), thus decoupling devices (stingers) are generally adopted to mitigate this issue. Several stinger configurations were then analyzed, in order to reduce inertia effects caused by the exciter moving part. A stinger is a commonly adopted component which allows to transmit the force along the exciter direction only, which is commonly measured by a load cell. All the other force and torque components should be filtered out by the stinger, so that the actual force acting on the component is really close to the one measured by the load cell. A simple test bench was equipped to study the performance of four different stinger configurations: rigid connection, beam stinger, wire stinger, and ball stinger.

\section{Electromagnetic shaker design}

Severe design specifications were required for the electromagnetic exciter, as reported in Table 1.

\begin{tabular}{ll}
\hline Quantity & Value \\
\hline Frequency range & $1-10 \mathrm{kHz}$ \\
Maximum radius & $17 \mathrm{~mm}$ \\
Peak force (at $10 \mathrm{kHz})$ & $0.1 \mathrm{~N}$ \\
\hline
\end{tabular}

Table 1. Electromagnetic excitation device main specifications, Bertini et al. (2015).

Even though several commercial solutions are available which satisfy frequency and force specifications, none of them were small enough to fulfill the geometrical constraint. For this reason, a custom solution was studied. A simple design configuration was chosen, having a fixed part and a moving part. A copper coil was wired on a support (fixed part), which was axially aligned with a floating permanent magnet (moving part). The permanent magnet guarantees high force density, so that the moving part volume is limited, thus reducing sizing and weight of the device. Neodymium magnets are available in a great variety of dimensions and at reasonable costs, so they were chosen for the present application. This choice also allowed to develop simple analytical/numerical models of the exciter, so a geometric optimization could be obtained. Aluminum alloy was chosen to machine all the structural parts. Since this material is nonferromagnetic, it does not influence the generated magnetic field. Other non-ferromagnetic and non-conductive materials were also considered (such as Plexiglas) with the aim to reduce eddy current losses. Anyway, machining issues could arise for these materials, introducing some limitations on the possible manufacturing geometries, so they were not chosen at the present research stage. For this reason, eddy current phenomenon may produce some overheating during operation and also require a higher power supply for the device. Experimental tests performed on prototypes showed that these effects were tolerable in the present application. Finally, the connection between the moving and the fixed parts was designed. The connection should guarantee a largely compliant support along the axial direction, while a higher stiffness was required along the radial direction.
This was achieved by using a rubber membrane, as reported below.

\section{Analytical models}

Solenoid-permanent magnet interaction is widely reported in literature and several different models are proposed Furlani (2001); Ravaud et al. (2010); Mapother and Snyder (1955); Robertson et al. (2011, 2012). In particular, two models were considered in this research. The first and simpler was based on Biot-Savart law, which allows to determine the flux density $\vec{B}$ on the axis of a current loop. The law was applied to the excitation device by modeling the solenoid as a cylinder, having the coil winded between inner and outer radii ( $r_{1}$ and $r_{2}$ respectively). A partial cylindrical portion of the coil was firstly considered, having $z$ as axial coordinate and a radial extension of $\mathrm{d} r$. The number of coils contained in each $\mathrm{d} z$ segment was supposed to be equal to $N /\left(L\left(r_{2}-r_{1}\right)\right) \mathrm{d} z \mathrm{~d} r$, being $L$ the total solenoid length and $N$ the total number of coils. The resultant intensity of $\vec{B}$ could be computed at any coordinate $z$ as the integral of the contributions of each portion $\mathrm{d} A=\mathrm{d} z \mathrm{~d} r$, from $r_{1}$ to $r_{2}$ and from $-L / 2$ to $L / 2$, being the origin of the reference system positioned at the center of the solenoid. The value of $|\vec{B}|$ was assumed to be constant along the radius, having a variation along the $z$ coordinate only, so that the computation could be limited to the solenoid axis only. The error introduced by this hypothesis decreases as the ratio between the length $L$ and the radius $r_{2}$ increases. The permanent magnet was modeled as a cylinder having a radius $R_{\mathrm{m}}$, a height $h$ and a constant magnetization $M$. According to the described model, the magnetic field in the solenoid could be computed Mapother and Snyder (1955) and then the total force acting on the magnet could be evaluated Furlani (2001):

$$
\begin{array}{r}
B_{1}=(z+L / 2) \ln \left(\frac{r_{2}+\sqrt{r_{2}^{2}+(z+L / 2)^{2}}}{r_{1}+\sqrt{r_{1}^{2}+(z+L / 2)^{2}}}\right) \\
B_{2}=(z-L / 2) \ln \left(\frac{r_{2}+\sqrt{r_{2}^{2}+(z-L / 2)^{2}}}{r_{1}+\sqrt{r_{1}^{2}+(z-L / 2)^{2}}}\right) \\
B_{z}(z)=|\vec{B}(z)|=\frac{\mu_{0} I N}{2 L\left(r_{2}-r_{1}\right)}\left(B_{1}-B_{2}\right) \\
F(Z)=\frac{\pi R_{\mathrm{m}}^{2} M}{\mu_{0}}\left(B_{z}(Z+h / 2)-B_{z}(Z-h / 2)\right)
\end{array}
$$

where $Z$ represents the distance between the center of the permanent magnet and the center of the solenoid, $\mu_{0}$ is the vacuum permeability and $I$ is the supplied current circulating in the solenoid.

In order to increase the reliability of the estimation, a second model was developed for comparison purposes. This model was based on the so called "shell method". The solenoid was modeled as a series of thin cylinders, where the current density is $I / N_{\mathrm{r}}$, being $N_{\mathrm{r}}$ the number of windings along the radial direction. The permanent magnet was modeled as a cylindrical thin surface too. This surface was characterized by a current density which value was related to the value of $M$. It was possible to compute the total force acting on the permanent magnet by summing the contributions $F_{S}$ determined by all the thin surfaces of the solenoid: 


$$
F(Z)=\frac{1}{N_{\mathrm{r}}} \sum_{n=1}^{N_{\mathrm{r}}} F_{S}\left(R_{\mathrm{m}}, r_{n}, h, L, Z\right)
$$

where $r_{n}$ represents the radial distance between the $n$-th thin surface and the axis of the permanent magnet. The formulation of $F_{S}$ involves complete elliptic integrals of the first, second and third kinds, and it is reported in Robertson et al. (2011, 2012).

Both the described models were implemented in an electronic sheet, providing very similar results in terms of force estimation, Figure 3. The models were further validated by performing an experimental test (see below): these results are also reported in the figure.

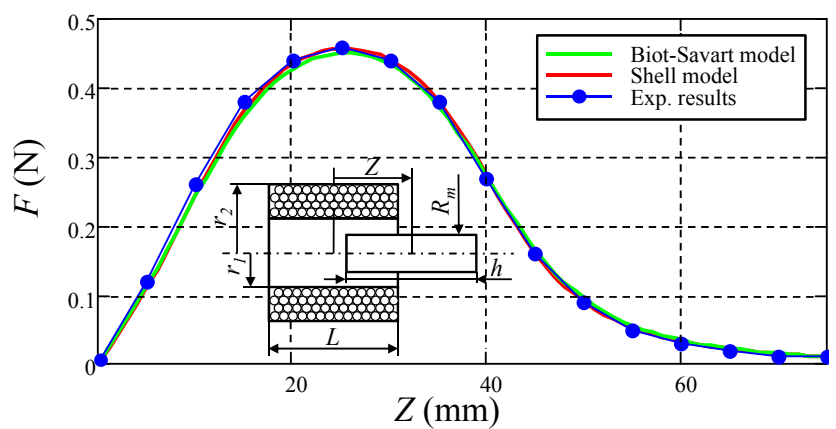

Figure 3. Analytical models comparison with $L / r_{1}=5$ and successful experimental validation.

Once the reliability of the models was proved, they could be used for optimization purposes, tuning the geometrical parameters in order to maximize the force intensity still respecting sizing limitations. Since some dimensions were fixed as reported in Table 1, the free parameters were represented by the permanent magnet dimensions and the total length of the solenoid $L$. The final optimal dimensions are reported in Table 2.

\begin{tabular}{ll}
\hline Quantity & Value \\
\hline$r_{1}$ & $10 \mathrm{~mm}$ \\
$r_{2}$ & $15 \mathrm{~mm}$ \\
$L$ & $50 \mathrm{~mm}$ \\
Wire diam. & $1.2 \mathrm{~mm}$ \\
$N$ & 150 \\
$h$ & $30 \mathrm{~mm}$ \\
$R_{\mathrm{m}}$ & $5 \mathrm{~mm}$ \\
\hline
\end{tabular}

Table 2. Electromagnetic shaker optimal dimensions.

\section{Moving part support}

The moving part support is a key component in the shaker design. Depending on the chosen stinger (see below) different preload conditions may be needed during operation. In particular, no preload is needed if a beam stinger is used, a tensile preload is needed if a wire stinger is used and a compression preload is needed if a ball stinger is used. The moving part support must then be able to provide a static support to the permanent magnet and also to carry the desired preload. Moreover, it should be almost insensitive to high frequency excitation, in order not to influence the shaker vibrational behavior. All these features were obtained by using an elastic membrane. A rubber membrane disk was glued to the outer diameter of the fixed part of the shaker during the assembly. In this way, the moving part was supported as almost free-free Ewins (2000) along the axial direction, i.e. no axial constraint was present. Thanks to the membrane properties, the radial stiffness was significantly higher than the axial stiffness, providing stable static support for any shaker mounting orientation. On the other hand, this solution introduced a new constraint on the shaker sizing. The support axial/radial stiffness highly depends on the unglued area of the membrane, so the clearance between the outer diameter of the magnet and the inner diameter of the coil support could not be decreased freely. Figure 4 shows a scheme of the moving part elastic support. In the actual device, the permanent magnet was made by separate cylinders, which are connected to each other through reciprocal magnetic forces. The outer magnetic disks allowed to attach the inner magnetic cylinder to the elastic membrane. This solution allowed to achieve the connection between the moving and the fixed part of the shaker without any fastener, which would be really difficult to fit in such a compact design. Moreover, the proposed solution allowed to apply both tensile or compression preload to the excited structure, so that all the available stingers could be used.

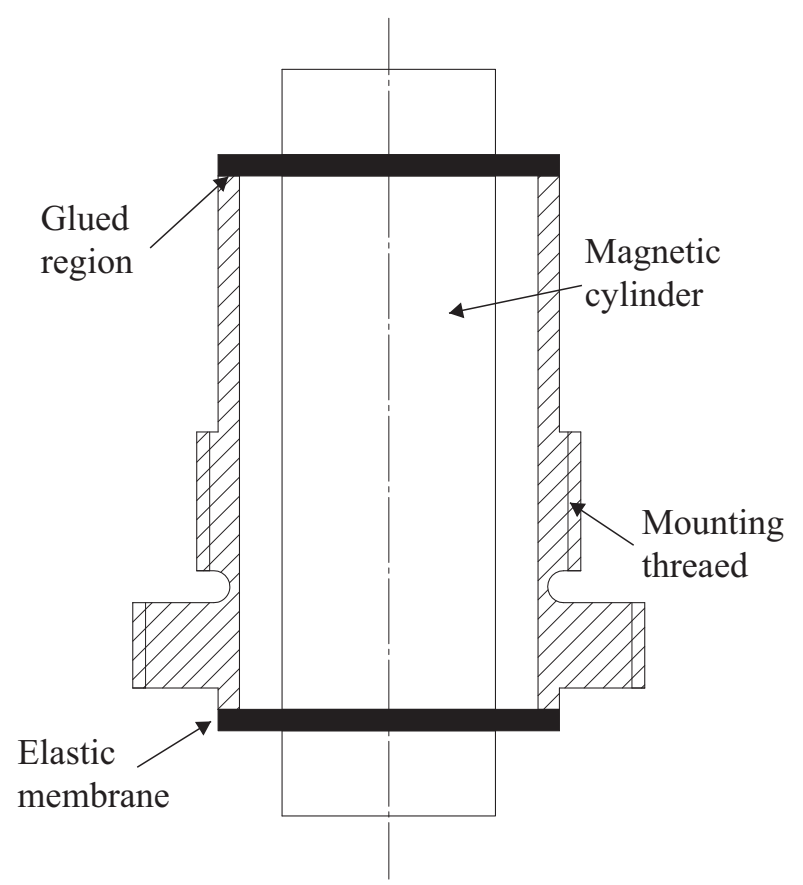

Figure 4. Elastic support schematic section view.

\section{Shaker tests}

The aforementioned analytical models allowed to optimize the dimensions of the shaker to satisfy the specifications as reported in Table 1. Anyway, the models estimate the interaction force between the copper coil and the permanent magnet, which corresponds to the force acting on the excited structure only in static conditions. A static test was firstly performed on a shaker prototype, Figure 5 (a). During the test, the permanent magnet was mounted on a load cell 
and the coil was moved along its axial direction in order to change the value of $Z$. The coil was supplied with a constant 2 A current, so the force intensity only depended on the axial position of the coil. The results are reported in Figure 3, which shows a successful validation of the proposed analytical models.

On the other hand, when the coil is supplied with a time varying current, the force acting on the target decreases with respect to the force acting on the permanent magnet (i.e. the force estimated by the analytical models). In particular, the difference between these two forces equals the product between acceleration and mass of the shaker moving part. For this reason, also a dynamic test was needed to ensure that the force intensity specification was fulfilled also at the highest working frequency of $10 \mathrm{kHz}$ (which implies the highest acceleration). The test setup involved a fixed support to hold the shaker and a rigid structure as a target structure. The target structure was a steel cantilever beam, having a cross section of $60 \times 100$, which was obviously remarkably stiffer than the shaker parts. The moving part of the shaker was attached to the target beam through a load cell, using a magnetic disk as shown in Figure 5 (b). No stinger was used during this test, in order to decouple the shaker behavior from other effects. The control signal was generated through a LMS SCADAS device, in terms of voltage amplitude and frequency. The power signal was supplied by a Behringer EP2000 amplifier. The proposed setup only provided an upper bound of the force intensity at any frequency. The actual available force during operation of course depends on the stiffness of the excited structure. Anyway, even if more compliant structures would cause a force reduction, they would also need a lower load to produce measurable displacements. Thus, the proposed test was considered to provide a reference value for the force intensity.

During the test, supplied voltage and current values, along with the generated force, were recorded in order to check both mechanical and electrical properties of the shaker. The diameter of the copper wire was $1.2 \mathrm{~mm}$, so the maximum supply current was limited to $10 \mathrm{~A}$. The chosen copper wire could undertake such high current levels with no damage only for a few minutes. Anyway, this limitation was not really sever for most working conditions, since about $10 \mathrm{~A}$ were needed to produce $0.14 \mathrm{~N}$ (more than the required $0.1 \mathrm{~N}$ ) only at $10 \mathrm{kHz}$. This showed that shaker overheating may be a potential warning only for really high frequency long lasting tests. Since long time excitation was not required during HRA test conditions, the result was considered acceptable.

\section{Stingers comparison}

Once the designed shaker proven to fulfill all the specifications, different stinger solutions were compared in order to choose the most suitable for the application. A stinger should ideally transmit just one force component along a chosen direction, perfectly filtering out the other five components (i.e. 3 torques and 2 forces). To prove the effectiveness of the considered stingers a specific test bench was setup as shown in Figure 6. A cantilever beam specimen was attached to two different shakers along the transverse orthogonal directions (i.e. vertical and horizontal). The stinger to be tested was mounted on the load cell

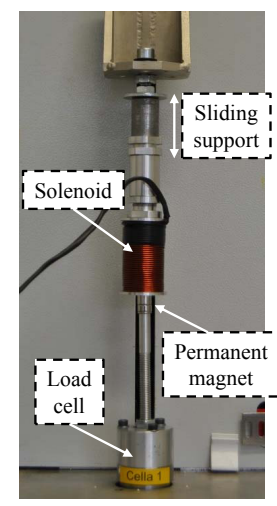

(a)

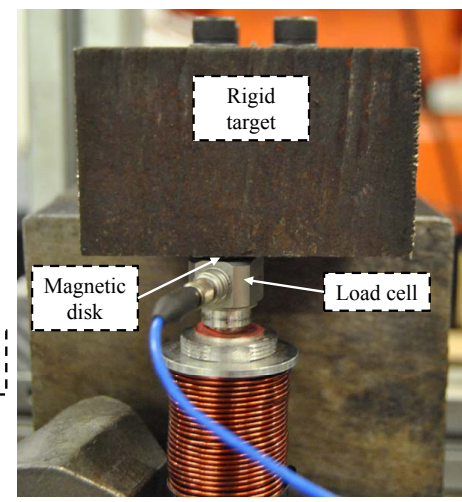

(b)
Figure 5. Shaker tests: (a) static test and (b) dynamic test.

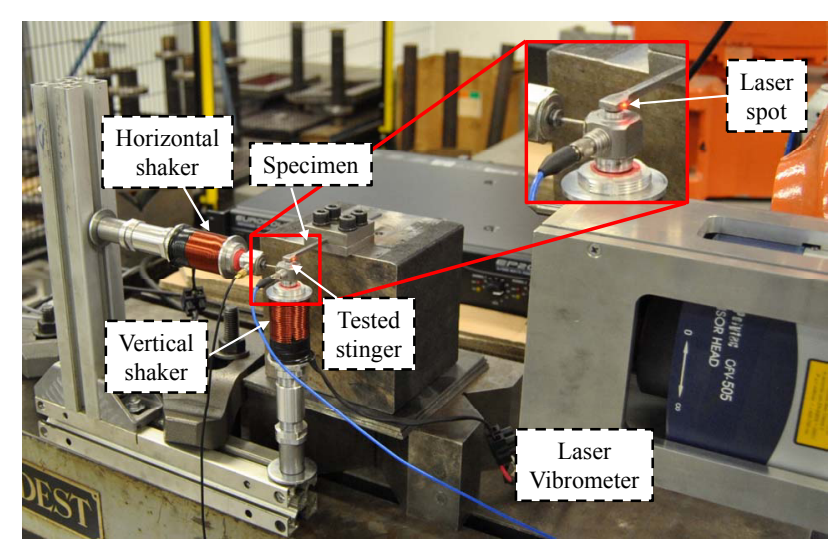

Figure 6. Test bench setup for stinger characterization.

of the vertical shaker, and then it was connected to the specimen along the vertical direction. The horizontal shaker was connected to the vertical load cell through a beam stinger and another load cell along the horizontal direction. The oscillation velocity of the cantilever beam was measured through a laser Doppler vibrometer. A preliminary test was performed by powering the vertical shaker only and measuring the vertical vibration of the specimen. The measured FRF (along vertical direction) highlighted that all the stingers show only a peak in correspondence of the specimen natural mode, proving to have an adequate axial response. Then, the transverse filtering test was performed. The vertical shaker was not powered during the test, but it was used only as a support to provide the required preload (tensile or compression). Only the horizontal shaker was powered during the test with a white noise voltage signal. In this way, the Frequency Response Function (FRF) between the force and the vibration velocity along the horizontal direction (measured by the laser) could be computed. If the stinger was able to efficiently filter out the undesired transverse load (low transverse stiffness), a really low FRF is expected. On the other hand, a not-zero FRF would indicate a stiffer and less efficient stinger behavior.

A reference FRF was obtained by gluing the vertical load cell to the specimen using no stinger at all. The tests were then repeated with a beam stinger, a wire stinger and a ball stinger supported with a conical or a spherical slot. The beam stinger represents the classical solution in dynamic 
analysis, while the wire stinger represents an evolution of the beam stinger with a lower transverse stiffness (this latter solution is less often adopted for the practical inconvenience of applying the tensile preload). Both these solutions require a fixed connection between the exciter and the measured structure. On the other hand, the ball stinger solution has a point-contact at one or both sides of the sphere (for conical and spherical slot respectively), which produces a single or double hinge connection between the exciter and the structure. Thus, the transverse stiffness of the connection is drastically reduced. The dimensions of the stingers were chosen to reduce mass and bending stiffness, thus enhancing transverse filtering capabilities, and considering practical limitations and commercial availability. The beam stinger required no preload $(\phi=1 \mathrm{~mm}, l=10)$, the wire stinger required tensile preload $(\phi=0.2 \mathrm{~mm}, l=7 \mathrm{~mm})$ and the ball stinger required a compression preload $(\phi=3 \mathrm{~mm})$. This latter solution presented some stability problems due to the compression preload. A stable equilibrium was achieved trying two different shaped supports, one having a conical slot and one having a spherical slot to host the ball. The first three considered stingers are shown in Figure 7, while the ball stinger mounting is shown in Figure 8, along with a schematic section view of the two proposed supports. All the stingers were screwed to the load cell and glued to the specimen, except the ball stinger which is kept in contact with the specimen through the compression preload. This represents an advantages for the ball stinger solution, as it reduces mounting time and increases connection reliability. On the other hand, Figure 7 shows that relevant mounting issues were found for the wire stinger solution, since the alignment of the wire with the shaker axis was not trivial. Moreover, the fragility of the wire also arose reliability limitations.

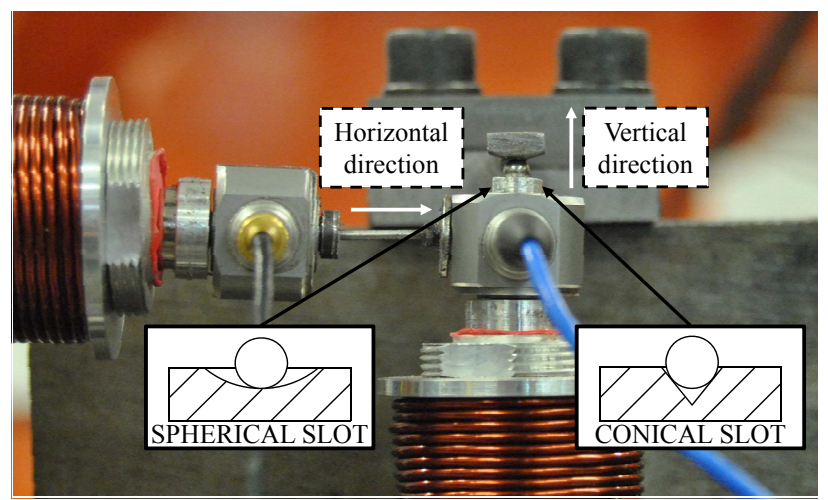

Figure 8. Ball stinger setup.

A comparison between all the FRFs obtained during the tests is reported in Figure 9. A peak close to $5500 \mathrm{~Hz}$ was found in all the FRFs, corresponding to a resonance frequency of the excited specimen. One or more peaks at frequencies lower than $4000 \mathrm{~Hz}$ were also found for the glue, the wire stinger, the beam stinger and the ball stinger supported with the conical slot. On the other hand, the ball stinger supported with the spherical slot provided an almost zero FRF in the whole frequency range $(1-10 \mathrm{kHz})$, showing only the peak close to $5500 \mathrm{~Hz}$. This experimental evidence confirmed that the ball stinger with the spherical slot provided the best decoupling along transverse direction, and thus the best transverse load filtering. These results suggested to choose the ball stinger with the spherical slot support as the best solution, at least in the studied range of load intensity and frequency.

\section{Conclusions}

This paper presents the design of a custom dynamic exciter for experimental harmonic response analysis tests. This device should present really small lateral dimensions $(<$ $34 \mathrm{~mm}$ ), operating in a wide frequency range $(1-10$ $\mathrm{kHz}$ ). Contactless electromagnets were firstly considered by designing a prototype having U-shaped ferromagnetic core and two copper windings on its arms. An analytical model was developed to predict the device behavior, and experimental tests were performed to evaluate the intensity of each load harmonic in the studied frequency range. This solution was found to be not suitable for the application. In particular, electromagnets showed to produce pretty low force level presenting control issues in the load harmonic content. Moreover, this solution could be used only to excite ferromagnetic components. Finally, a direct measurement of the force was not possible with contactless solutions, so the actual load acting on the component could not be precisely measured.

The focus then moved to a classic contact shaker solution. The available commercial solutions did not guarantee the specifications fulfillment, so a custom shaker was designed for the application. A solenoidpermanent magnet configuration was chosen, fulfilling all the imposed specifications. The optimization was achieved by implementing analytical and numerical models. The influence of the excitation device on the studied structure was investigated and several different stinger configurations were compared: glue, beam, wire and ball stinger. Two different shaped mounting supports were tested for the ball stinger. The effectiveness of these stingers was studied through a dedicated test bench, and the ball stinger with the spherical slot mounting support provided the best transverse load filtering performances. This stinger configuration also was the more convenient in terms of mounting time and connection reliability.

\section{References}

Battiato G, Firrone C and Berruti T (2017) Forced response of rotating bladed disks: Blade tip-timing measurements. Mechanical Systems and Signal Processing 85: 912-926. DOI: 10.1016/j.ymssp.2016.09.019.

Berruti T, Firrone C and Gola M (2011) A test rig for noncontact traveling wave excitation of a bladed disk with underplatform dampers. Journal of Engineering for Gas Turbines and Power 133(3): 032502. Doi: 10.1115/1.4002100.

Bertini L, Monelli B, Neri P, Santus C and Guglielmo A (2014a) Robot assisted modal analysis on a stationary bladed wheel. In: Proceedings of ASME 2014 12th Biennial Conference on Engineering Systems Design and Analysis (ESDA), Copenhagen, Denmark. pp. 1-8. DOI:10.1115/ ESDA2014-20636. 


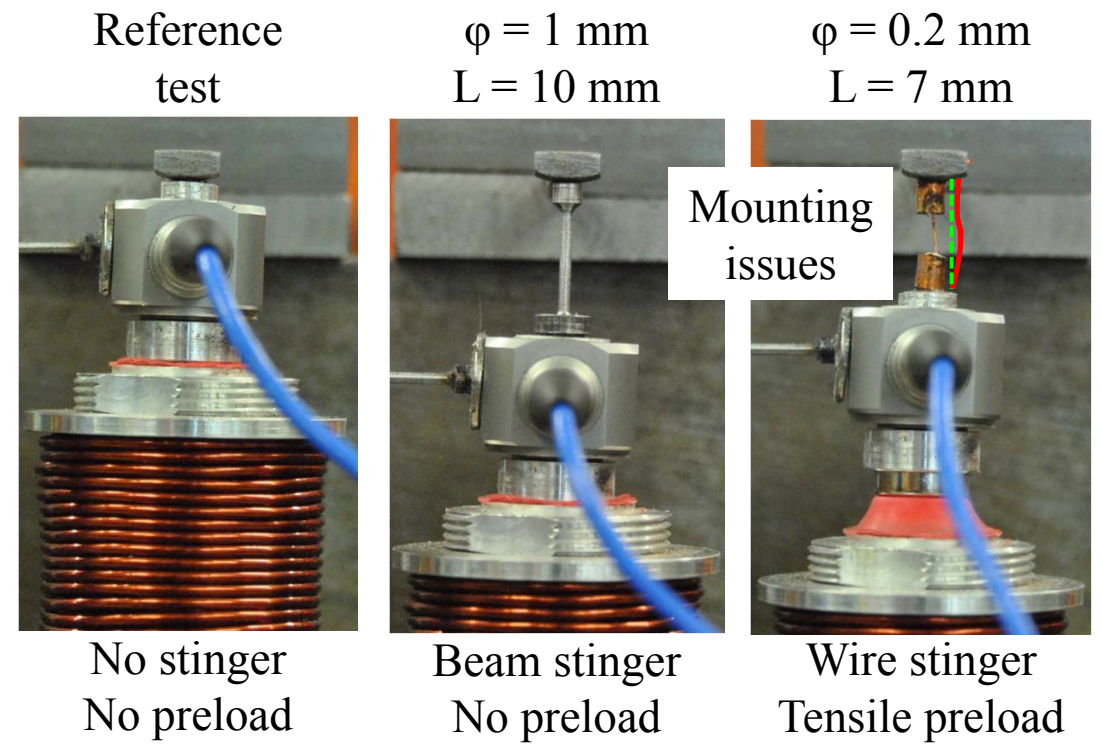

Figure 7. Glue, beam and wire stinger setup.

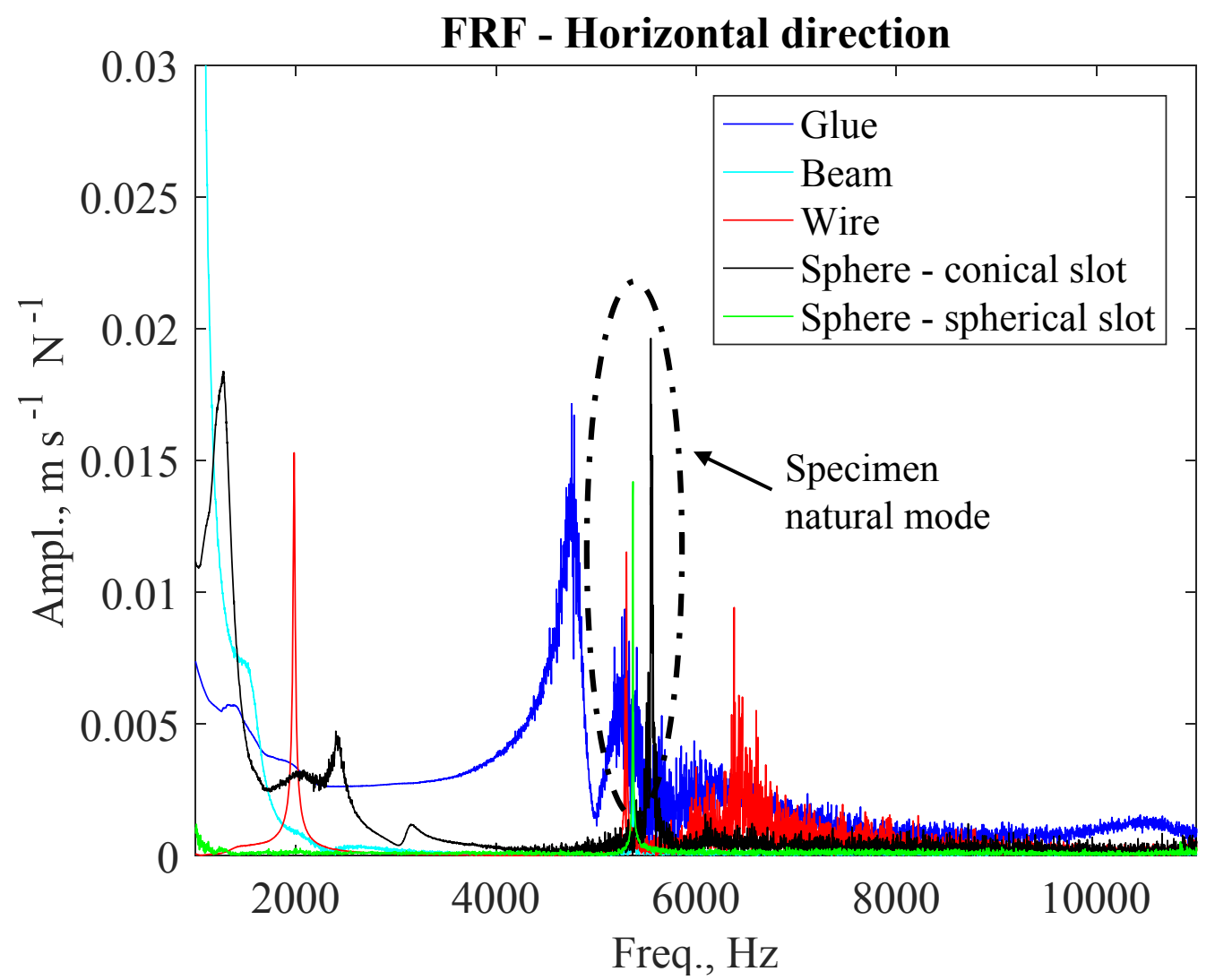

Figure 9. Test results, FRF along the horizontal direction.

Bertini L, Neri P and Santus C (2015) Design and optimization of a compact high-frequency electromagnetic shaker. In: Proceedings of 11th International Conference on Engineering Vibration, ICoEV 2015. pp. 1-9. Ljubljana, Slovenia, 7-10 September.

Bertini L, Neri P, Santus C and Guglielmo A (2016) Automated experimental modal analysis of bladed wheels with an anthropomorphic robotic station. Experimental Mechanics 57(2): 1-13. Doi: 10.1007/s11340-016-0223-5.
Bertini L, Neri P, Santus C and Guglielmo A (2017) One exciter per sector test bench for bladed wheels harmonic response analysis. In: Proceedings of the ASME 2017 Turbomachinery Technical Conference \& Exposition. pp. 1-9.

Bertini L, Neri P, Santus C, Guglielmo A and Mariotti G (2014b) Analytical investigation of the safe diagram for bladed wheels, numerical and experimental validation. Journal of Sound and Vibration 333: 4771-4788. Doi: 10.1016/j.jsv.2014.04.061. 
Bidaut Y and Baumann U (2012) Identification of eigenmodes and determination of the dynamical behavior of open impellers. In: Proceedings of ASME Turbo Expo, Parts A and B, Copenhagen, Denmark. pp. 1063-1073.

Ewins D (2000) Modal Testing, theory, practice and application, 2nd Edition. Research Studies Press Ltd - Wiley, Hertfordshire, England.

Firrone C and Berruti T (2012) An electromagnetic system for the non-contact excitation of bladed disks. Experimental Mechanics 52: 447-459. DOI:10.1007/s11340-011-9504-1.

Firrone C, Berruti T and Gola M (2013) On force control of an engine order-type excitation applied to a bladed disk with underplatform dampers. Journal of Vibration and Acoustics 135: 1-9. DOI:10.1115/1.4023899.

Furlani E (2001) Permanent Magnet and Electromechanical Devices. Academic Press - Elsevier, London, England.

Jones K and Cross C (2003) Traveling wave excitation system for bladed disks. Journal of propulsion and power 19: 135-141.

Kammerer A and Abhari R (2009) Experimental Study on Impeller Blade Vibration During Resonance - Part I: Blade Vibration Due to Inlet Flow Distortion. Journal of Engineering for Gas Turbines and Power 131(2): art. no. 022508. Doi: 10.1115/1.2968869.

Mapother D and Snyder J (1955) The axial variation of the magnetic field in solenoids of finite thickness. Technical report, University of Illinois Bulletin, Urbana, Illinois.

Martarelli M, Castellini P, Santolini C and Tomasini E (2011) Laser doppler vibrometry on rotating structures in coastdown: resonance frequencies and operational deflection shape characterization. MEASUREMENT SCIENCE AND TECHNOLOGY 22: 1-16. DOI:10.1088/0957-0233/22/11/ 115106.

Maschio TB (2012) Experimental investigation on the forced response of a dummy counter-rotating turbine stage with friction damping. Journal of Engineering for Gas Turbines and Power 134(122502): 1-8. DOI:10.1115/1.4007325.

Mukhopadhyay N, Chowdhury SG, Das G, Chattoraj I, Das S and Bhattacharya D (1998) An investigation of the failure of low pressure steam turbine blades. Engineering Failure Analysis 5(3): 181-193. Doi: 10.1016/S1350-6307(98)00016-8.

Neri P (2013) Studio della forza di interazione tra campo magnetico e bersaglio ferromagnetico mediante prove sperimentali e modelli analitici e numerici. In: Proceedings of the 42th Convegno Nazionale AIAS. pp. 1-10.

Neri P (2017a) Bladed wheels damage detection through nonharmonic fourier analysis improved algorithm. Mechanical Systems and Signal Processing 88: 1-8. Doi: 10.1016/j.ymssp.2016.11.010.

Neri P (2017b) Interaction force between magnetic field and ferromagnetic target: Analytical, numerical and experimental study. Submitted to Applied and Computational Mechanics .

Neri P and Peeters B (2015) Non-harmonic fourier analysis for bladed wheels damage detection. Journal of Sound and Vibration 356: 181-194. Doi: 10.1016/j.jsv.2015.06.048.

Oberholster A and Heyns P (2009) Online condition monitoring of axial-flow turbomachinery blades using rotor-axial eulerian laser doppler vibrometry. Mechanical Systems and Signal Processing 23(5): 1634-1643. Doi: 10.1016/j.ymssp.2009.01.001

Pesek L, Vanek F, Bula V and Cibulka J (2011) Excitation of blade vibration under rotation by synchronous electromagnet.
Engineering MECHANICS 18(3/4): 249-257.

Ravaud R, Lemarquand G, Babic S, Lemarquand V and Akyel C (2010) Cylindrical magnets and coils: Fields, forces, and inductances. IEEE TRANSACTIONS ON MAGNETICS 46: 3585-3590. DOI:10.1109/TMAG.2010.2049026.

Rieger N (1990) The high cost of failure of rotating equipment. In: Proceedings of the 44th Meeting of the Mechanical Failures Prevention Group. pp. 1-17. AD-A226 670.

Robertson W, Cazzolato B and Zander A (2011) A simplified force equation for coaxial cylindrical magnets and thin coils. IEEE TRANSACTIONS ON MAGNETICS 47: 2045-2049. DOI: 10.1109/TMAG.2011.2129524.

Robertson W, Cazzolato B and Zander A (2012) Axial force between a thick coil and a cylindrical permanent magnet: Optimizing the geometry of an electromagnetic actuator. IEEE TRANSACTIONS ON MAGNETICS 48: 2479-2487. DOI: 10.1109/TMAG.2012.2194789.

Singh M, Matthews T and Ramsey C (1994) Fatigue Damage of Steam Turbine Blade Caused by Frequency Shift Due to Solid Buildup - A Case Study. In: Proceedings of the 23rd Turbomachinery Symposium. pp. 107-114.

Singh M and Vargo J (1989) Reliability Evaluation of Shrouded Blading Using the SAFE Interference Diagram. Journal of Engineering for Gas Turbines and Power 111(4): 601-609. Doi: 10.1115/1.3240296. 